\title{
Identification of a novel p53-dependent activation pathway of STAT1 by antitumour genotoxic agents
}

\author{
I Youlyouz-Marfak ${ }^{1}$, N Gachard ${ }^{1}$, C Le Clorennec ${ }^{1}$, I Najjar ${ }^{2}$, F Baran-Marszak ${ }^{3}$, L Reminieras ${ }^{4}$, E May ${ }^{5}$, GW Bornkamm ${ }^{6}$, R Fagard $^{2}$ \\ and $\mathrm{J}$ Feuillard ${ }^{\star 1}$
}

Chemotherapeutic drugs such as fludarabine ${ }^{*}$, doxorubicin or cisplatin are very potent activators of the anti-oncogene p53. Convergent studies suggest that p53 and STAT1 (signal transducer and activator of transcription 1) cooperate in the induction of cell death. We show that these drugs are also activators of STAT1 in p53-expressing cells, but not in p53-null cells. STAT1 activation was obtained in the presence of both the secretion inhibitor brefeldine $A$ and the inhibitor of RNA synthesis, actinomycin D. p53-dependent STAT1 activation was reversed by overexpression of MDM2 and siRNAs against p53. Genetic analysis of p53 showed that expression of transcriptionally inactive p53 punctual mutants markedly increased Y701-STAT1 phosphorylation, and suggests that the p53 DNA-binding domain was alternatively involved in STAT1 activation or p53 multimerization. Immunoprecipitation experiments showed that ataxia telangiectasia mutated, p53, STAT1 and c-Abl1 (Abelson murine leukaemia viral oncogene homologue 1) were associated together. Treatment of cells with the c-Abl1 tyrosine kinase inhibitor STI571 decreased STAT1 activation by genotoxic drugs. Finally, genotoxic agents sensitized cells in response to very low doses of both interferon $\alpha$ and $\gamma$ (IFN $\alpha$ and $\gamma$ ). These results show that genotoxic drugs induce STAT1 activation, an effect that depends on p53 protein but not on p53 transcriptional activity, and point to a novel pathway of STAT1 activation by genotoxic drugs, with involvement of c-Abl1 tyrosine kinase in sensitizing cells to IFN response.

Cell Death and Differentiation (2008) 15, 376-385; doi:10.1038/sj.cdd.4402270; published online 9 November 2007

Among the drugs used for treatment of cancers, genotoxic agents such as fludarabine ${ }^{*}$, doxorubicin (Dx) or cisplatin are widely used as inhibitor of DNA synthesis, crosslinking DNA or intercalating agents, respectively. These drugs are very potent activators of p53, a transcription factor able to block the cell cycle and to induce the apoptotic programme (May and May ${ }^{1}$ for review). In addition to its transcriptional activity, p53 interacts directly or indirectly with other transcription factors such as c-myc, the nuclear factor kappa B (NF- $k \mathrm{~B})$ subunit RelA ${ }^{2}$ or STAT1 (signal transducer and activator of transcription 1). ${ }^{3,4}$ It is noteworthy that both STAT1 and p53 share various target genes involved in cell cycle arrest or in apoptosis such as $\mathrm{p} 21^{5}$ and CD95. ${ }^{6}$ The cytotoxic activity of p53 inducers such as fludarabine* is increased by STAT1 transcriptional activation. ${ }^{4}$ Physical interaction between STAT1 and p53 is associated with a negative regulation of MDM2 expression and with an increase in p53 activation. ${ }^{3}$

STAT1 is essential for signalling by interferons (IFNs). IFN $\gamma$ is a specific activator of STAT1, resulting in its phosphorylation on tyrosine 701 (pY701-STAT1) and on serine 727 (pS727-STAT1). Other kinases have been shown to be able to phosphorylate STATs such as epidermal growth factor (EGF) and platelet-derived growth factor (PDGF) tyrosine kinase receptors or the $\mathrm{c}$-Abl1 (Abelson murine leukaemia viral oncogene homologue 1 ) oncogene. ${ }^{7}$ STAT1 protein exists in two forms, an active isoform, STAT $1 \alpha$, and a naturally inhibitory truncated form, STAT1 $\beta$. Even if predominantly cytosolic, latent unphosphorylated STAT1 is constantly shuttling between the cytosol and the nucleus via a mechanism that involves both the nucleoporin $153 \mathrm{kDa}$ (Nup153) and the exportin chromosome region maintenance 1 (CRM1).,

Activation of STAT1 is now considered as a pro-apoptotic event. $^{6,10,11}$ STAT1 activation is usually associated with a decrease in proliferation rate and an increase in apoptosis. Activation of STAT1 is required for downregulation of c-myc and cyclins in leukemic cells treated with all-trans retinoic acid. $^{12}$ STAT1 deletion accelerates tumour development in p53-null mice. ${ }^{13}$ Through activation of STAT1 homodimers, IFN $\gamma$ regulates genes involved in the immune response such as TAP1, MHC class II, IRF1 (interferon regulatory factor 1); $\mathrm{PKR}$, in the regulation of the cell cycle, such as induction of p21 and p $27^{5}$ or repression of c-myc ${ }^{14}$ and in the apoptosis,

\footnotetext{
${ }^{1}$ Centre National de la Recherche Scientifique, UMR CNRS 6101, Faculté de Médecine de Limoges, Université de Limoges, CHU Dupuytren, Laboratoire d'Hématologie, Limoges, France, Equipe labellisée de la Ligue Nationale Contre le Cancer; ${ }^{2}$ UPRES EA 3406, Université Paris 13, Service de Biochimie, Hôpital Avicenne, Assistance Publique Hôpitaux de Paris, Bobigny, France; ${ }^{3}$ Service d'Hématologie Biologique, Hôpital Avicenne, Assistance Publique Hôpitaux de Paris, Bobigny, France; ${ }^{4}$ Service d'Hématologie Clinique, CHU Dupuytren, Limoges, France; ${ }^{5} \mathrm{FRE}$ CNRS 2937, Institut André Lwoff, France and ${ }^{6} \mathrm{GSF}$, Institute of Clinical Molecular Biology and Tumour Genetics, 81377 Munich, Germany

${ }^{*}$ Corresponding author: J Feuillard, Laboratoire d'Hématologie et UMR CNRS 6101, CNRS, Faculté de Médecine, Université de Limoges, CHU Dupuytren, 2 Avenue Martin Luther King, Limoges 87042, France. Tel: + 3355505 61 80; Fax: 3355505 61 85; E-mail: jean.feuillard@chu-limoges.fr

Keywords: STAT1; p53; c-Abl1; apoptosis; cancer; chemotoxic drugs; ATM

Abbreviations: ATM, ataxia telangiectasia mutated; BFA, brefeldin A; C-Abl1, Abelson murine leukaemia viral oncogene homologue 1; CRM1, chromosome region maintenance 1; Dx, doxorubicin; IFN, interferon; IRF1, interferon regulatory factor 1; JAK, Janus protein tyrosine kinase; NF- $\kappa$ B, nuclear factor kappa B; Nup153, nucleoporin $153 \mathrm{kDa}$; PARP, poly-ADP ribose polymerase; PDGF, platelet-derived growth factor; STAT1, signal transducer and activator of transcription; TRAIL, tumour necrosis factor-related apoptosis inducing ligand; VEGF, vascular epithelial growth factor

Received 16.4.07; revised 13.9.07; accepted 01.10.07; Edited by RA Knight; published online 09.11.07
} 
such as FAS, FAS-L, PML1, tumour necrosis factor-related apoptosis-inducing ligand (TRAIL), IRF1 or caspase $3 .^{15}$

Therefore, convergent studies suggest that p53 and STAT1 cooperate in the induction of apoptosis, and that STAT1 favours p53 activation. However, to our knowledge, the role of p53 in STAT1 activation is not documented. Our results suggest that genotoxic agents such as fludarabine*, Dx or cisplatin are STAT1 activators. This STAT1 activation depends on the presence of p53 protein and is modulated by the tyrosine kinase inhibitor STI571 with involvement of the c-Abl1 kinase. Moreover, activation of STAT1 by genotoxic agents sensitizes cells to IFN response in induction of apoptosis.

\section{Results}

Genotoxic agents induce STAT1 activation. We previously showed that inhibition of STAT1 activation blocks p53-dependent cell cycle arrest by fludarabine* 4 and Townsend et al. ${ }^{3,16}$ showed that STAT1 and p53 cooperate together in induction of apoptosis. These results led us to raise the question of STAT1 activation by genotoxic agents. We treated BL2 cells, a p53 wild-type (p53wt) Burkitt cell line, with three different p53 inducers: fludarabine ${ }^{*}$, Dx and cisplatin. The activity of these reagents was assessed by the western blot detection of the serine 15 phosphorylated form of p53 (pS15-p53) (Figure 1a). In this cell line, treatment with any of these three reagents resulted in phosphorylation of tyrosine 701 of STAT1 (pY701-STAT1) (Figure 1a). We also noticed weak but detectable levels of pY701-STAT1 in untreated cells.

To determine whether Y701-STAT1 phosphorylation induced by genotoxic agents depends on p53 transcriptional activity, we treated the BL41 cell line, a cell line with a p53 inactive mutation at arginine codon 248 (p53 $\left.{ }_{\mathrm{R} 248 \mathrm{Q}}\right),{ }^{17}$ with these three genotoxic agents. Like in BL2 cells, weak levels of pY701-STAT1 were found in untreated BL41 cells. As a second significant result, we found that fludarabine*, Dx and cisplatin induced STAT1 activation in $p 53_{\mathrm{R} 248 \mathrm{Q}}$ BL41 cells (Figure 1a). As expected, ${ }^{17}$ BL41 cells showed low levels of p53 transcriptional activity, as assessed with a p21 promoter luciferase reporter gene (not shown). These genotoxic agents did induce STAT1 transcriptional activity in BL41 cells (Figure 1b), as well as enhanced mRNA levels of endogenous STAT1 target genes such as TAP1 or IRF1 (Figure 1c).

Y701-STAT1 phosphorylation by genotoxic agents is not related to an autocrine effect. We then wanted to eliminate the hypothesis of STAT1 activation by soluble factors such as IFNs. We could not assess the IFN activity of the supernatant of our cell cultures because it would be contaminated with the genotoxic agents used to activate STAT1. Thus, we used indirect methods that consisted in blocking mRNA synthesis or cytokine secretion. We first used actinomycin $\mathrm{D}$, a well-known agent able to both block mRNA synthesis and to induce genotoxic stress and p53 activation. ${ }^{18}$ We found that actinomycin D induced Y701STAT1 phosphorylation after $1 \mathrm{~h}$ treatment (Figure $2 \mathrm{a}$ ). Meanwhile, mRNA levels of p21, a target gene of both p53 and STAT1, were significantly decreased after actinomycin D treatment (Figure 2b), in agreement with inhibition of mRNA synthesis by this agent. Quantitative RT-PCR assays showed that basal levels of IFN $\gamma$ mRNA were barely detectable (cycle threshold (CT) over 33), and no induction of IFN mRNA was found after actinomycin $D$ treatment (Figure $2 \mathrm{~b}$ for IFN $\gamma$, and not shown for IFN $\alpha$ and $\beta$ ).
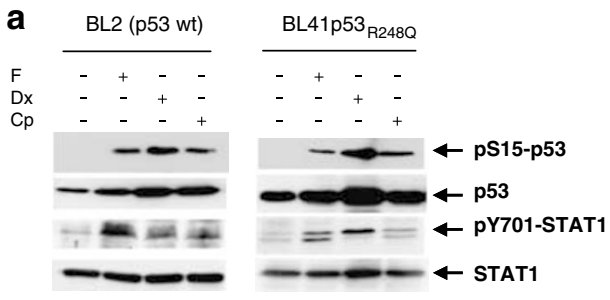

b
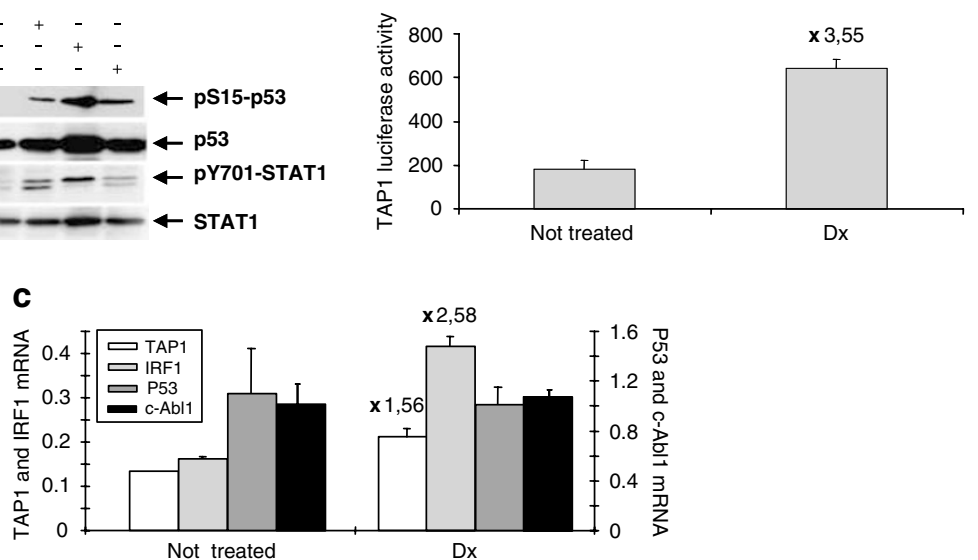

Figure 1 Induction of Y701-STAT1 activation by genotoxic agents. (a) Induction of Y701-STAT1 phosphorylation. Two million BL2 or BL41 cells were treated with either $30 \mu \mathrm{M}$ fludarabine $(\mathrm{F})^{*}, 1 \mu \mathrm{M}$ doxorubicin (Dx) or $30 \mu \mathrm{M}$ cisplatin (Cp) for $6 \mathrm{~h}$. Detection of pS15-p53, p53, pY701-STAT1 and STAT1 proteins was performed by western blot as described in Materials and Methods (b) Induction of STAT1 transcriptional activity. Five million BL41 cells were transfected with the TAP1 promoter luciferase reporter gene vector and co-transfected with the pRLTK control vector expressing the Renilla gene. Transfected cells were treated with Dx or without Dx (not treated) for $16 \mathrm{~h}$. TAP1 promoter activity was defined as the ratio between luciferase and the Renilla activities (c) Induction of STAT1 target genes. mRNA expression of the STAT1 target genes TAP1 and IRF1, as well as p53 and c-Abl1 as control, was assessed by real-time quantitative RT-PCR as described in Materials and Methods. BL 41 cells were treated with Dx or without Dx (not treated) for $12 \mathrm{~h}$. For TAP1, IRF1 and p53, mRNA levels were normalized to those of c-Abl1 gene and to the RNA pool (see Materials and Methods). For c-Abl1, results were normalized to the RNA pool only. Legends are presented within the graphic. Fold changes are indicated at the top of each column of the histogram for TAP1 and IRF1. These figures are representative of at least three independent experiments 
a

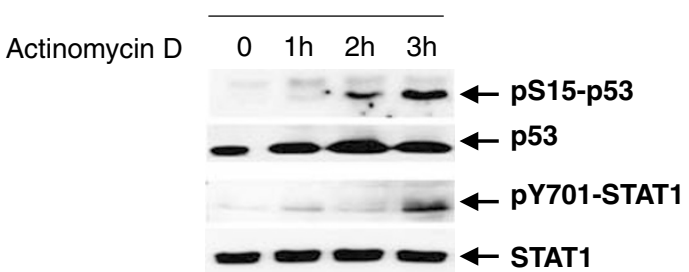

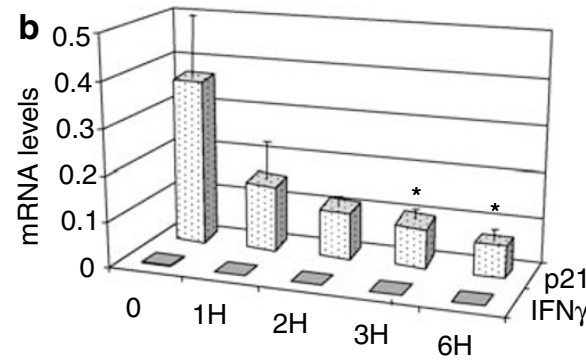

C

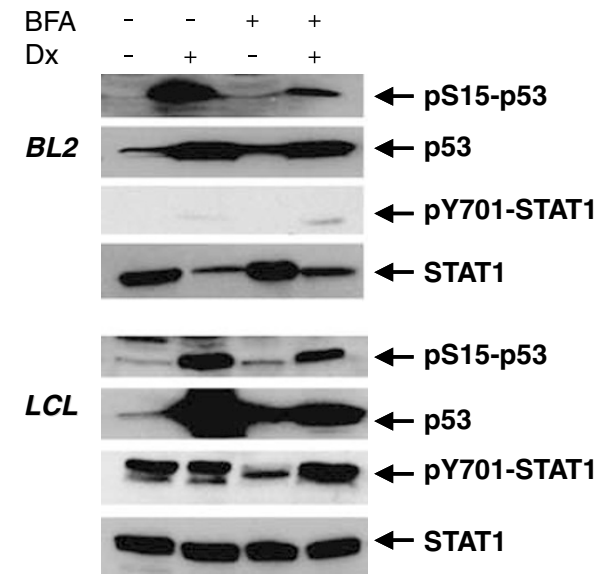

Figure 2 Induction of Y701-STAT1 phosphorylation by genotoxic agents in the absence of transcriptional activity and secretion. (a) Expression of pS15-p53, p53, pY701STAT1 and STAT1 protein in BL2 cells treated with the mRNA synthesis inhibitor actinomycin D. Time course of actinomycin D treatment is indicated at the top of each lane (b) Kinetics of IFN $\gamma$ and p21 mRNA expression, quantified by RT-QPCR, in BL2 cells treated with actinomycin D (statistical differences between treated and untreated cells are shown by an asterisk) (c) Western blot detection of pS15-p53, p53, pY701-STAT1 and STAT1 in BL2 cells (upper panel) or PRI LCL cells (lower panel) treated with (+ ) or without (-) brefeldin A (BFA; $5 \mathrm{~h}$ pre-incubation) and/or doxorubicin (Dx, 16 h incubation). Results presented are representative of at least three independent experiments

Treatment of p53wt BL2 cells with the inhibitor of cytokine secretion, brefeldin A (BFA), did not block STAT1 activation by fludarabine* (not shown) or Dx (Figure 2c). This was confirmed in PRI cells, a p53wt LCL cell line in which STAT1 activation is related to autocrine secretion of IFN $\gamma .{ }^{19}$ As expected in this cell line, BFA markedly decreased STAT1 autocrine activation (Figure $2 \mathrm{c}$ and Najjar et al. ${ }^{19}$ ). Treatment of PRI cells with fludarabine* (not shown) or Dx induced Y701-STAT1 phosphorylation in the presence of BFA.

Altogether, these results clearly suggest that activation of STAT1 by genotoxic agents is not due to the transcription and the secretion of a soluble factor.

Activation of STAT1 by genotoxic agents depends on p53 protein. Since STAT1 and p53 may interact with each other, ${ }^{3,4}$ we wanted to assess the role of p53 protein in STAT1 activation by genotoxic agents. Figure $3 a$ shows that Dx did not induce Y701-STAT1 phosphorylation in HL60 or in Jurkat cells, two p53-null cell lines. ${ }^{20}$ We transiently transfected p53-null HL60 cells with a pcDNA vector coding for p53wt or for three different p53 transcriptionally inactive variants, $p 53_{\mathrm{R} 248 \mathrm{Q}}, \mathrm{p} 53_{\mathrm{R} 156 \mathrm{P}}$ and $\mathrm{p} 53_{\mathrm{R} 273 \mathrm{H}^{21,22}}$ (Figure $3 \mathrm{~b}$ ). As previously shown, restoration of p53 expression was associated with its auto-activation. ${ }^{1}$ Transfection of either p53wt or mutated p53 variants induced Y701-STAT1 phosphorylation (Figure 3b). Co-transfection of p53wt and its inhibitor MDM2 expression vector in HL60 cells resulted in a decrease in Y701-STAT1 phosphorylation (Figure 3c).
Results presented in Figure $3 \mathrm{~b}$ and $\mathrm{c}$ show that Dx treatment hardly increased Y701-STAT1 phosphorylation after p53 transfection in HL60 cells. Confirming results in HL60 cells, p53 ${ }_{\mathrm{R} 248 \mathrm{Q}}$ siRNA transfection of BL41 cells resulted in a decrease of both $\mathrm{p} 53_{\mathrm{R} 248 \mathrm{Q}}$ protein expression and STAT1 activation by $\mathrm{Dx}$, whereas transfection of these cells with p53wt siRNA or control siRNA had no significant effect on both p53 protein levels and STAT1 activation by Dx (Figure 3d).

Altogether, this series of results clearly demonstrates that overexpression of p53 at least was associated with Y701STAT1 phosphorylation. Of note, it was not possible to conclude from these experiments on the role of p53 activation, since p53 transfection was associated with its constitutive phosphorylation on serine 15 and since Dx treatment did not increase pS15-p53 or pY701-STAT1 levels in these conditions.

Relationship between p53 structure and activation of STAT1. We next sought to correlate STAT1 activation with p53 structure. To answer this question, we used a series of deletion mutants of p53 in addition to the three mutated p53 variants already used (Figure 4a). p53 del11-69 lost the $\mathrm{N}$-terminal activation domain targeted by ATM (ataxia telangiectasia mutated)/ATR. p53 trunc291 lost both the Cterminal regulatory and the oligomerization domain (OD) with its hinge region, $\mathrm{p} 53_{\text {trunc } 355}$ lost the $\mathrm{C}$-terminal regulatory domain, $\mathrm{p5} 3_{\text {trunc338 }}$ was also partly deleted in the 

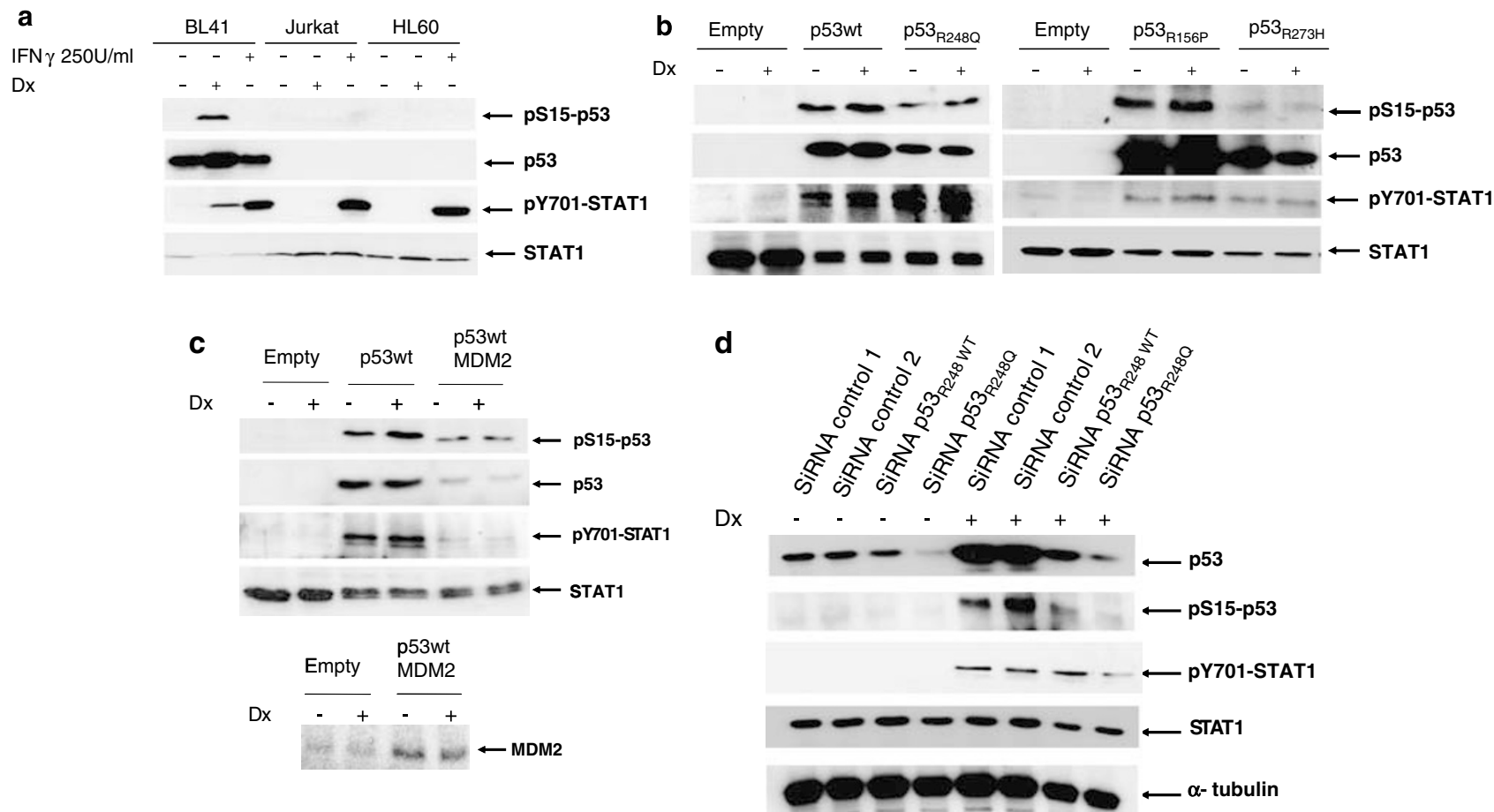

Figure 3 Relationship between p53 protein expression and induction of Y701-STAT1 phosphorylation by genotoxic agents. Western blot detection of pS15-p53, p53, pY701-STAT1 and STAT1 is presented in (a-d) MDM2 detection in (c) and tubulin detection in (d). (a) BL41, HL60 and Jurkat cells were treated with (+) or without (-) either doxorubicin for $6 \mathrm{~h}(\mathrm{Dx})$ or IFN $\gamma$ for $1 \mathrm{~h}$ (b) HL60 cells (p53 null) were transiently transfected either with an empty or a p53-expressing vector coding for p53wt, p53 ${ }_{\mathrm{R} 248 \mathrm{Q}}$, p53 $3_{\mathrm{R} 156 \mathrm{P}}$ or $\mathrm{p} 53_{\mathrm{R} 273}$. Then, transfected cells were treated with $(+)$ or without $(-) \mathrm{Dx}$ for $6 \mathrm{~h}(\mathbf{c}) \mathrm{HL} 60$ cells were transfected either with the empty vector or with the p53wt vector or co-transfected with both p53wt and MDM2 vectors and treated with $(+)$ or without $(-)$ Dx for $6 \mathrm{~h}$. Western blot detection of pS15-p53, p53, pY701-STAT1 and STAT1 are shown in the upper panel. Western blot detection of MDM2 is shown in the lower panel (d) BL41 cells were transfected with either control 1, control 2, p53 ${ }_{\text {R248WT }}$ (p53wt) or p53 $3_{\mathrm{R} 248 \mathrm{Q}}$ siRNA at $1 \mu \mathrm{g} / 5$ million cells. Transfected cells were treated with $(+)$ or without $(-)$ Dx for $6 \mathrm{~h}$. Tubulin expression was tested as a control not affected by siRNA transfection. For each experiment, transfection with a control pCMV-enhanced green fluorescent protein coding vector showed that more than $70 \%$ of cells were transfected. These figures are representative of at least three independent experiments

oligomerization domain, p53 del11-69_trunc338 was deleted for both the $\mathrm{N}$-terminal activation domain and $\mathrm{C}$-terminal end from amino acid 339. p53 transcriptional activity was compared with the corresponding STAT1 transcriptional activity of the same extracts (Figure $4 \mathrm{~b}$ ). p53 protein levels were systematically checked from whole cellular extracts for each experiment (Figure $3 \mathrm{~b}$ and not shown). As expected, transfection of p53wt induced both p53 and STAT1 transcriptional activity. The three $p 53_{\mathrm{R} 248 \mathrm{Q}}, \mathrm{p} 53_{\mathrm{R} 156 \mathrm{P}}$ and p53 ${ }_{\mathrm{R} 273 \mathrm{H}}$ mutants exhibited marked decrease in p53 transcriptional activity and a moderate decrease in STAT1 transcriptional activity. p53 del11-69 showed a complete loss of p53 transcriptional activity while levels of STAT1 activity were comparable to those of punctual mutants, suggesting that activation of $\mathrm{p} 53$ by its phosphorylation is not necessary for p53-dependent STAT1 activation. p53 ${ }_{\text {trunc291 }}$, a mutant unable to tetramerize, showed virtually no p53 activity and the highest levels of STAT1 activity. This suggests that neither the C-terminal nor the OD was involved in STAT1 activation by p53. Results with this mutant definitively confirm that STAT1 activation by p53 was not due to its transcriptional activity. Both p53 del11-69 and p53 $3_{\text {trunc291 }}$ mutants point the p53 DNA-binding domain as the domain required for p53-dependent STAT1 activation. Results with p53 trunc355 were exactly the opposite than with p53 $3_{\text {trunc291 }}$. Indeed, p53 $3_{\text {trunc355 }}$ exhibited the same transcriptional activity as p53wt, whereas STAT1 activity was completely abolished. Normal p53 transcriptional activity was expected for this mutant since it is constitutively under a tetramerization active form, due to the lack of the C-terminal regulatory domain. Interestingly, $\mathrm{p} 53_{\text {trunc338 }}$ with a partial deletion of the OD showed both a moderate decrease in p53 transcriptional activity and a moderate recovery in STAT1 activity. Thus, taken together the results from p53 $3_{\text {trunc291 }}, \mathrm{p} 53_{\text {trunc355 }}$ and p53 $3_{\text {trunc338 }}$ mutants indicate that tetramerization of p53 is not compatible with STAT1 activation. In other words, engagement of p53 DNA-binding domain in p53 tetramerization would prevent p53-dependent STAT1 activation.

STAT1, p53 and c-Abl1 are physically associated. Our results raised the question of which tyrosine kinase was responsible for STAT1 phosphorylation. The ATM serine kinase is thought to act as a DNA damage sensor. ATM is one of the major kinases responsible for the cascade of successive phosphorylation of p53, among which S15-p53 phosphorylation. ${ }^{23}$ Furthermore, it has been shown that ATM activates the tyrosine kinase c-Abl1 by phosphorylation on serine $465 .{ }^{24} \mathrm{C}-\mathrm{Abl} 1$ is one of the tyrosine kinases able to phosphorylate STAT1. ${ }^{25,26}$

We found that immunoprecipitation of ATM allowed to reveal STAT1 as a partner (Figure $5 \mathrm{a}$ ). This result led us to 

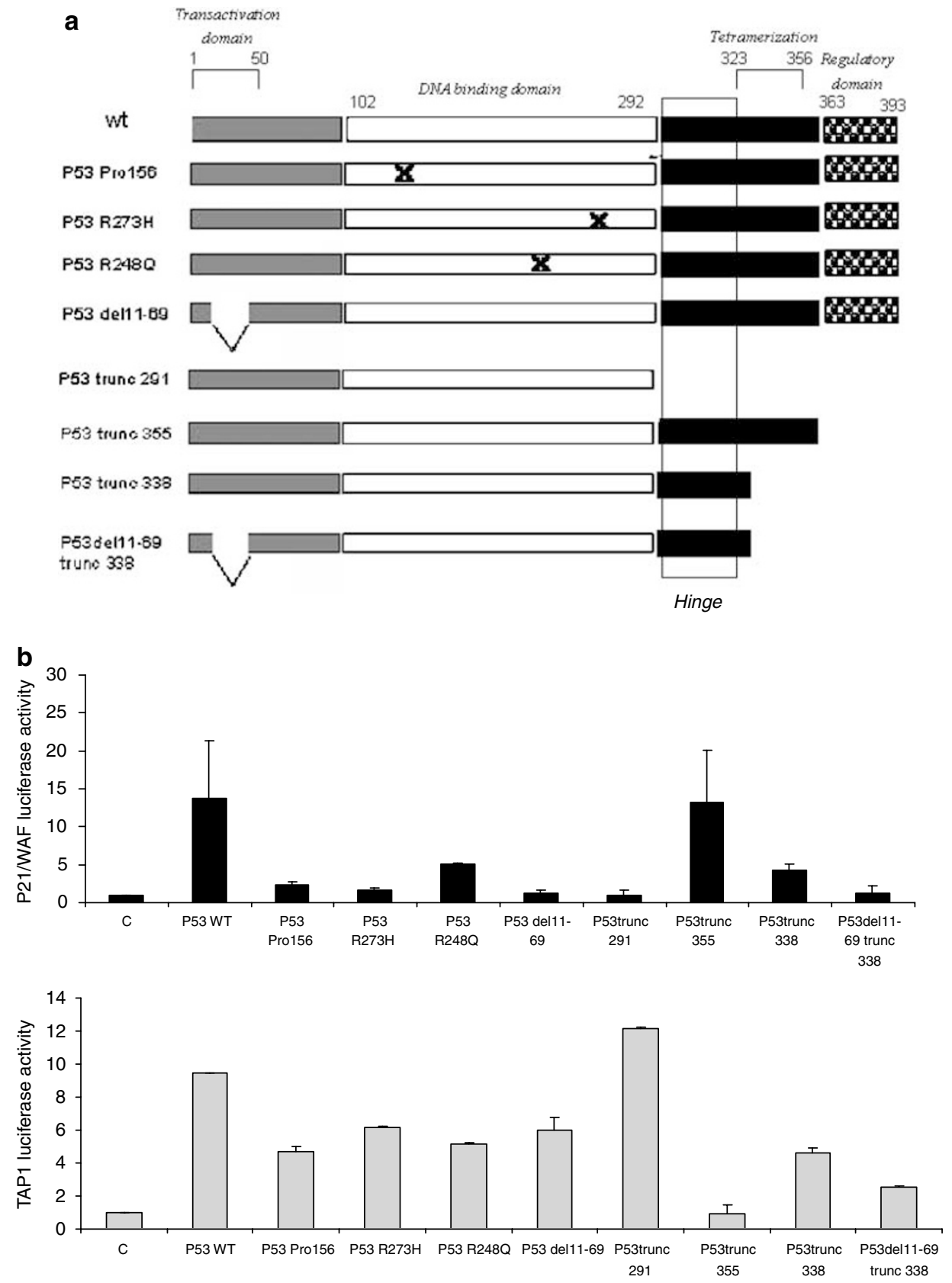

Figure 4 Genetic analysis of the relationships between p53 structure and STAT1 activation in p53-null HL60 cell line. (a) Schematic representation of the p53 mutants used in this study in comparison to the full-length p53wt (see text for description) (b) p53-null HL60 cells were co-transfected with different p53-expressing vectors listed in (a) and with both the pRLTK control vector expressing the Renilla gene and the luciferase reporter gene containing the human p21/WAF1-p53-responsive element (upper panel) or the human TAP1-STAT1-responsive element (lower panel). Luciferase assays were performed on extracts from cells collected $24 \mathrm{~h}$ following transfection. p21 and TAP1 promoter activity was defined as the ratio between luciferase and the Renilla activities. The values represent mean relative luciferase activity from three independent experiments

search for a direct association among p53, STAT1 and c-Abl1. Immunoprecipitation of p53 showed that it is physically associated with both STAT1 and c-Abl1, confirming previous studies (Figure 5b; Levav-Cohen et al. ${ }^{27}$ and Wei et al. ${ }^{28}$ ). Immunoprecipitation of c-Abl1 showed that STAT1 is physically associated with c-Abl1 and p53, and that this interaction is increased after Dx treatment of $p 53_{R 248 Q}$ BL41 cells. Reciprocal immunoprecipitation of STAT1 showed that both c-Abl1 and p53 physically interact with STAT1, an effect that is markedly increased by Dx treatment of BL41 cells. These results show that STAT1, p53 and c-Abl1 interact physically, at least two by two, and that this interaction is increased by Dx treatment of cells.

We next looked at cytosolic versus nuclear content of each of these partners following Dx treatment (Figure 5c). As expected, pS15-p53 was exclusively located in the nucleus after Dx treatment of BL41 cells. p53 whole protein was predominantly found in the nucleus, even if some cytosolic 

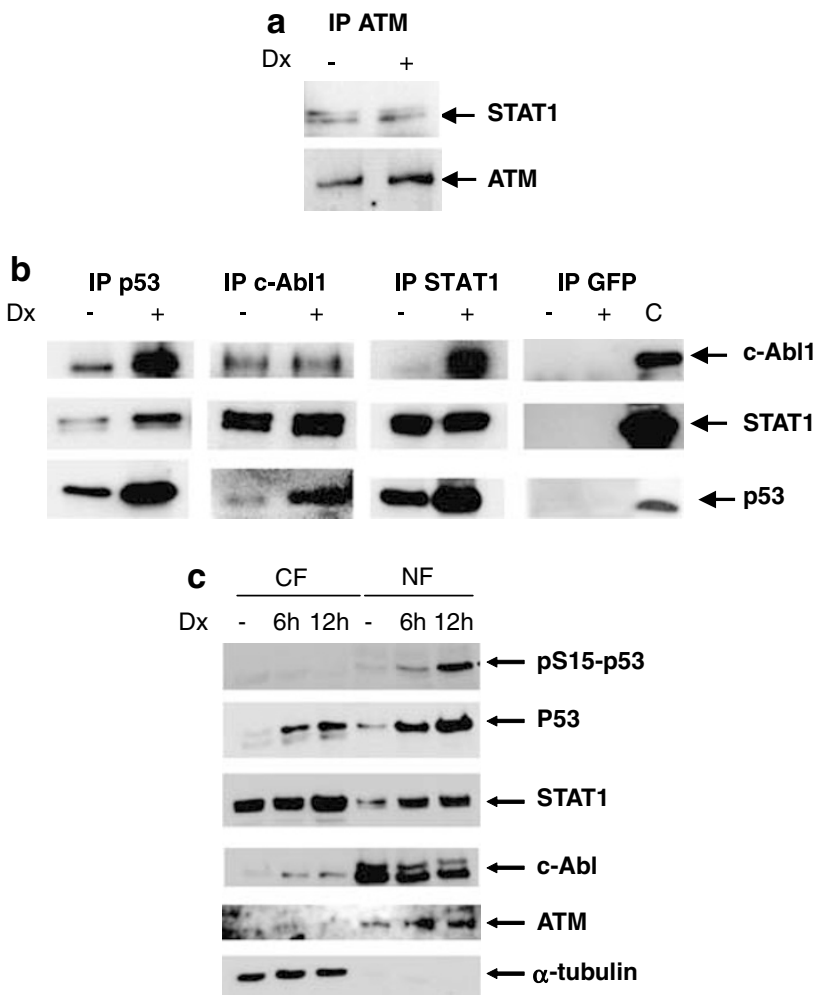

Figure 5 ATM, STAT1, p53 and c-Abl1 are physically associated with each other. BL41 cells were treated with $(+)$ and without $(-)$ Dx for $6 \mathrm{~h}(\mathrm{a}$ and $\mathrm{b})$ and $12 \mathrm{~h}$ (c). (a) Immunoprecipitation of ATM and revelation of STAT1 (b) Immunoprecipitation of p53, c-Abl1 and STAT1 followed by western blot revelation of these three proteins. Immunoprecipitation of irrelevant GFP was performed to control the specificity of western blot revelation of p53, c-Abl and STAT1. The lane $C$ corresponds to the western blot control (c) Cytosolic and nucleic extracts from BL41 cell line treated with Dx at the indicated time course (top of each lane) were submitted to western blot analysis for the indicated proteins

p53 could be found. Increased levels of p53 protein after Dx treatment was likely to be due to $p 53$ stabilization since Dx did not induce increase in p53 mRNA in these cells (Figure $5 \mathrm{c}$ ). STAT1 was predominantly located in the cytosol, even if some STAT1 protein was found in the nucleus in the absence of Dx treatment. Dx treatment of cells enhanced nuclear localization of STAT1, an additional indication that p53 activators are able to activate STAT1. c-Abl1 was predominantly found in the nucleus in untreated cells. Some c-Abl1 protein was found in the cytosol after Dx treatment. ATM was clearly found in the nucleus, and hardly detected in the cytosol. Dx treatment did not induce any detectable ATM nuclear export to cytosol. Thus, it is known from these results that p53, STAT1, c-Abl1 and ATM would coexist together in the nucleus mainly in untreated cells, and that this coexistence was reinforced by Dx treatment.

Y701-STAT1 phosphorylation after treatment with genotoxic agents is inhibited by the tyrosine kinase inhibitor STI571. Our results led us to test whether the tyrosine kinase c-Abl1 could be activated after treatment of cells with genotoxic agents. We used the STI571 agent, a tyrosine kinase inhibitor designed to block c-Abl1 tyrosine kinase activity of the chimeric Bcr-Abl1 product in chronic myeloid leukaemia (Ohno ${ }^{29}$ for review). Treatment of BL41 cells with STI571 markedly decreased the induction of STAT1 tyrosine 701 phosphorylation by both fludarabine* and Dx (Figure 6a). Furthermore, STI571 inhibited STAT1 activation in p53-null HL60 cell transfected with the p53wt expression vector (Figure 6b). As a control of c-Abl1 activation by Dx and its inactivation by STI571, we looked at phosphorylation of p73 on tyrosine 99, a target of c-Abl1, in p53-null HL60 cells. Dx did increase phosphorylation of p73, and STI571 did block this effect (Figure 6c). As a control of the specificity of STI571, levels of phosphorylated CD19 on tyrosine 513, a target of the lyn tyrosine kinase, remained unchanged after Dx and STI571 treatment (Figure 6d). We also found that treatment of these cells with IFN $\gamma$ in the presence of STI571 did not inhibit STAT1 phosphorylation, indicating clearly that this inhibitor did not inhibit Janus protein tyrosine (JAK) kinases (Figure 6e).

Altogether, these results suggest that c-Abl1 tyrosine kinase is involved in STAT1 activation following treatment of cells with $\mathrm{p} 53$ activators.

Dx sensitizes cells to effect of IFNs on apoptosis. To understand the role of STAT1 activation by genotoxic agents in proliferation and apoptosis in the context of transcriptionally inactive $p 53$, we pretreated $p 53_{\mathrm{R} 248 \mathrm{Q}}$ BL41 cells with Dx and treated them with the lowest dose of IFN $\alpha$ or $\gamma$ able to induce detectable Y701-STAT1 phosphorylation. Pretreatment of cells with Dx markedly increased activation of STAT1 by IFNs without modifying p53 phosphorylation (Figure 7a). Additionally, mRNA induction of the STAT1 target gene TAP1 by IFNs was markedly enhanced if cells were pretreated with Dx (Figure $7 \mathrm{~b}$ ). We then looked for induction poly-ADP ribose polymerase (PARP) cleavage as a result of induction of apoptosis transduction pathways. The three agents Dx, IFN $\alpha$ and $\gamma$ were able to induce PARP cleavage. This effect was markedly enhanced when cells were sensitized by Dx pretreatment. This synergistic effect between Dx and IFNs was also found for induction of CD95 at both protein and RNA levels (Figure 7c and not shown).

Altogether, these results suggest that genotoxic agents sensitize cells to IFN response for STAT1 activation and for induction of apoptosis.

\section{Discussion}

Relationships between STAT1 activation and p53 activators have been suggested by both Townsend et al. ${ }^{3}$ and McDermott et al., ${ }^{10}$ who showed that S727-STAT1 phosphorylation was increased by genotoxic agents. But, directed mutagenesis showed that STAT1 pY701 is the key activation event, allowing STAT1 dimerization, translocation to the nucleus and activation of target genes, ${ }^{7}$ while serine 727 phosphorylation facilitates the transcriptional activity of pY701-STAT1. Thus, our results clearly show for the first time that agents able to induce p53 activation are also able to induce concomitant STAT1 activation. Technically, it is of note that pY701-STAT1 is not stable and, at least in our hands, did not resist cellular fractionation. Only whole cellular extracts after instantaneous cell lysis could be analysed. Also, levels of Y701-STAT1 phosphorylation induced by genotoxic agents 

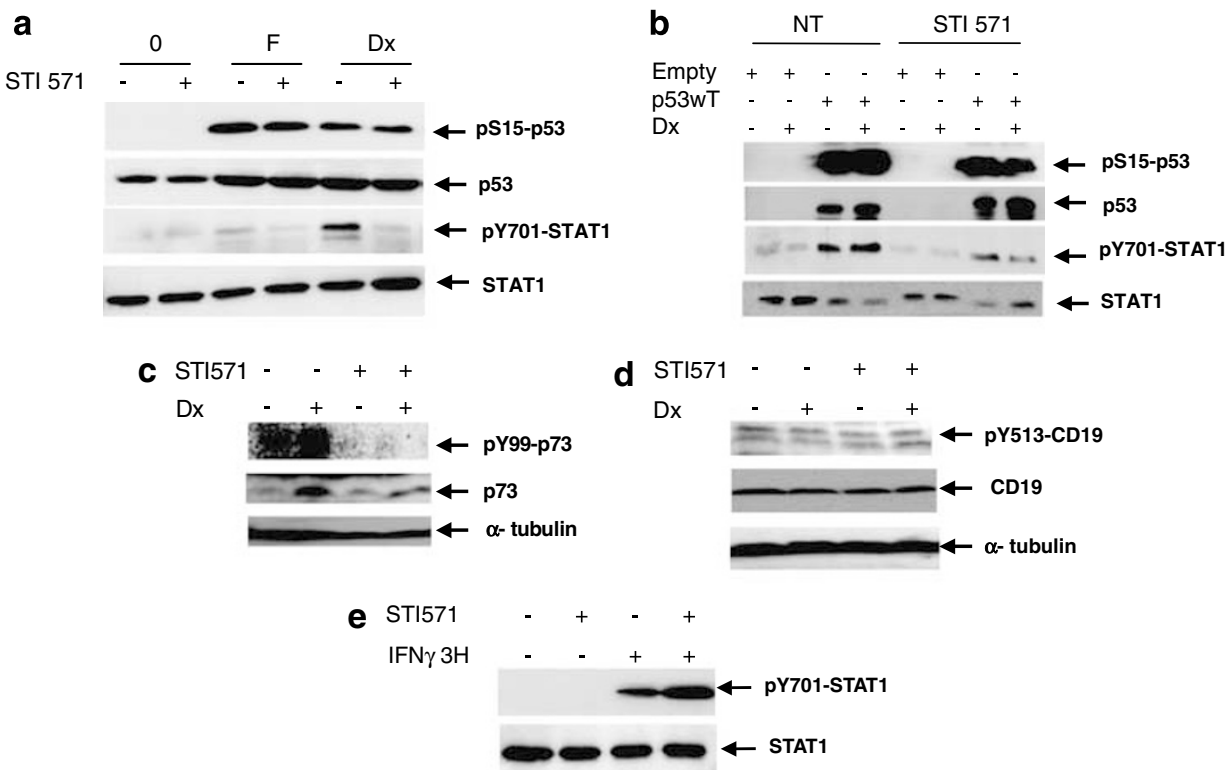

Figure 6 Effect of the tyrosine kinase inhibitor STI571 on the induction of Y701-STAT1 phosphorylation by fludarabine* and doxorubicin (Dx). (a) Effect of the tyrosine kinase inhibitor STI571 on phosphorylation of Y701-STAT1 on BL41 cells. P53 ${ }_{\mathrm{R} 248 \mathrm{Q}} \mathrm{BL} 41$ cells were pretreated with or without STI571 for $5 \mathrm{~h}$ and additionally treated with or without fludarabine* or Dx for $6 \mathrm{~h}$ (b) Effect of the tyrosine kinase inhibitor STI571 on phosphorylation of Y701-STAT1 on HL60 cells. STI571 indicates pretreatment of transfected cells for $5 \mathrm{~h}$ with this tyrosine kinase inhibitor. Dx indicates treatment of cells with doxorubicin for $6 \mathrm{~h}$, whereas NT indicates absence of pretreatment. $(+)$ and $(-)$ indicate whether cells were transfected with or without the empty vector (empty) or the p53wt coding vector (p53wt) or whether cells were treated with or without Dx (c) Effect of STI571 on the induction of Dx-induced Y99-p73 phosphorylation, as a target of c-Abl1 (d) Control of specificity of STI571 on phosphorylation of Y513-CD19 as irrelevant target of c-Abl1, CD19 (e) Control of STI571 effect on Y701-STAT1 phosphorylation by IFN $\gamma$. Detection of c-Abl1, pS15-p53, p53, pY701-STAT1, STAT1 and $\alpha$-tubulin was performed by western blot. Results presented are representative of at least three independent experiments

never reached those induced by standard experimental doses of IFNs (Figure 3a for example). To evidence STAT1 activation by genotoxic agents, we increased both gel loading (equivalent to $2 \times 10^{6}$ million cells rather than $0.5 \times 10^{6}$ ) and time exposure of the films. This made us able to detect some weak constitutive STAT1 activation in untreated BL2 and BL41 cells. This activation of STAT1 could be simply due to high levels of p53 expression in these cells.

Y701-STAT1 phosphorylation was induced by genotoxic agents in the presence of the secretion inhibitor BFA. Thus, it is unlikely that STAT1 activation by p53 activators could be related to secretion of soluble factors. Treatment of cells with actinomycin $D$, which is both a well-known inhibitor of RNA synthesis and an intercalating agent creating irreversible DNA damages, markedly induced Y701-STAT1 phosphorylation, thus making unlikely the hypothesis that Y701-STAT1 phosphorylation is related to transcriptional activity. We found no activation of STAT1 by genotoxic agents in p53-null cell lines. STAT1 activation was restored in these p53-null cell lines by expression of both p53wt and three different p53 transcriptionally inactive mutants. Inhibition of p53 activation by overexpression of MDM2 or after transfection of siRNA against p53 mRNA decreased Y701-STAT1 phosphorylation in p53-expressing cells. These results show for the first time that STAT1 activation by p53 activators depends on the presence of p53 protein but not on its transcriptional activity.

To understand the relationship between p53 and STAT1 activation, we used deletion mutants of the different domains of p53 protein. Our results show that the $\mathrm{N}$-terminal domain, the $\mathrm{C}$-terminal regulatory domain and the $\mathrm{OD}$ are dispensable for STAT1 activation. The results suggest a model according which p53-dependent STAT1 activation depends on p53 DNA-binding domain. The fact that constitutive engagement of p53 DNA-binding domain in p53 tetramerization prevents STAT1 activation and that both p53 and STAT1 interact physically with each other (Townsend et al., ${ }^{3}$ Baran-Marszak et al. ${ }^{4}$ and our results) strongly suggests that STAT1 interacts directly with the DNA-binding domain of p53.

ATM is a serine/threonine kinase belonging to the PI-3 kinase-related family, which is activated after DNA damages such as double-stranded breakpoints. ${ }^{30}$ In addition to p53 phosphorylation, ATM is involved in the serine phosphorylation of various proteins playing a pivotal role in cell proliferation and/or apoptosis, including the tyrosine kinase c-Abl1. ${ }^{24}$ Interactions between ATM and c-Abl1 may involve a multi-molecular complex with p53, resulting in stabilization of p53 tetramers. ${ }^{28}$ One of the downstream targets of c-Abl1 is STAT $1 .{ }^{25,26}$ It is noteworthy that, meanwhile the oncogenic properties of the chimeric $b c r-A b l 1$ gene are due to constitutive activity of the catalytic domain of $\mathrm{c}-\mathrm{Abl1}$, the native $c-A b / 1$ gene has been shown to induce cell growth arrest and promote apoptosis, ${ }^{31}$ an effect that is likely to be related to the stabilization of $p 53 .^{28}$ This led us to search for a relationship among ATM, p53, c-Abl1 and STAT1. We found that ATM is associated with STAT1, and that p53, c-Abl1 and STAT1 interact physically, at least two by two. Treatment of cells with the tyrosine kinase inhibitor STI571, a synthetic inhibitor blocking the catalytic activity of c-Abl1 and used in the treatment of CML, markedly decreased Y701-STAT1 phosphorylation by genotoxic agents. STI571 agent is active 

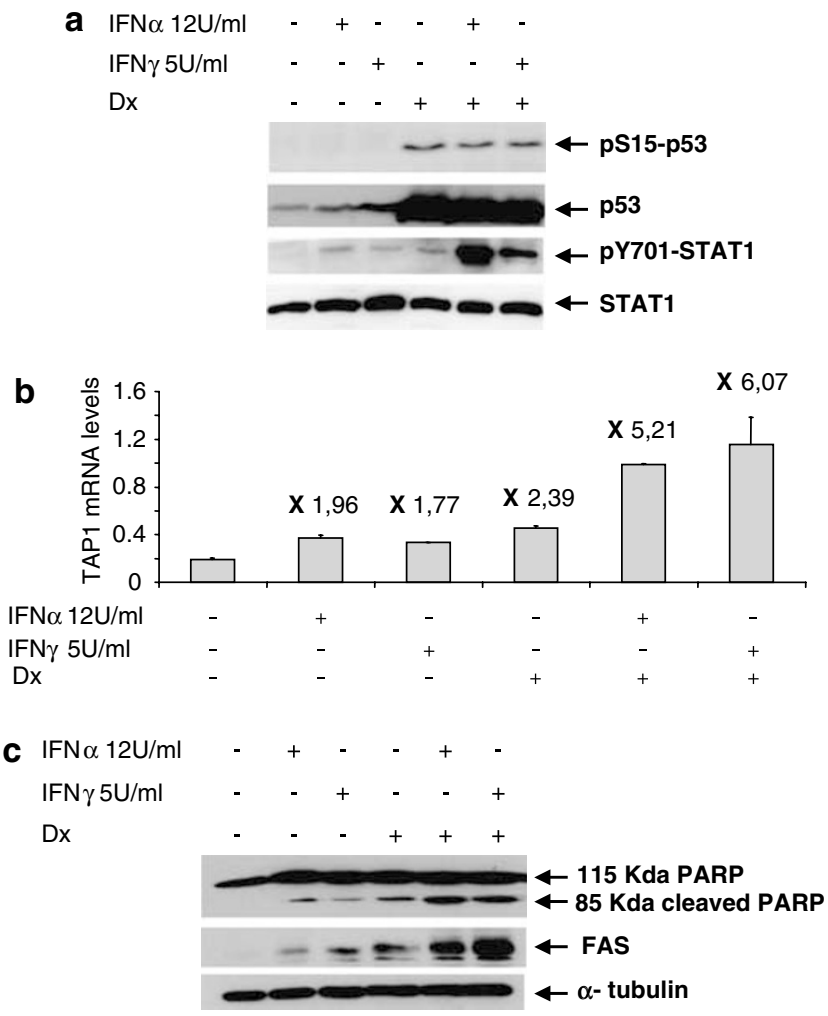

Figure 7 Doxorubicin (Dx) sensitizes cells to the effect of IFN $\alpha$ and $\gamma$. Cells were pretreated with Dx for $6 \mathrm{~h}$ and were then incubated with IFN $\alpha$ or $\gamma$ for $6 \mathrm{~h}$, at concentrations of 12 and $5 \mathrm{U} / \mathrm{ml}$, respectively, corresponding to the lowest doses able to activate detectable phosphorylation of Y701-STAT1. $(+)$ and $(-)$ indicate whether cells were treated with or without for each experimental conditions. Dx pretreatment enhanced IFN's induced Y701-STAT1 phosphorylation (a), expression of the STAT1 target gene TAP1 (b) and cleavage of PARP and CD95 expression (c)

against c-Abl1, c-Kit and PDGF or vascular epithelial growth factor (VEGF) receptors. ${ }^{32}$ Burkitt lymphoma cell lines, belonging to the B-cell lineage, do not express $\mathrm{C}-\mathrm{Kit}$, PDGF or VEGF receptors (M Schlee and $F$ Baran-Marszak, unpublished cDNA array results). In contrast, these cells express significant levels of c-Abl1 transcripts, as well as c-Abl1 protein. Taken together, our results suggest that c-Abl1 is a candidate tyrosine kinase for p53-dependent Y701STAT1 phosphorylation by genotoxic agents, and point to a novel original p53-dependent STAT1 activation pathway. The four proteins, STAT1 (including latent unactivated STAT1), p53, c-Abl1 and ATM may shuttle from nucleus to cytosol and from cytosol to nucleus, $8,33,34,31$ rendering theoretically possible their interaction in both compartments. Analysis of protein content of nuclear and cytosolic extracts favours the hypothesis of an intranuclear interaction among these partners. We propose a model according to which ATM would act as a platform allowing the positioning of the three different partners, p53, STAT1 and c-Abl1, each of them being necessary for p53-dependent STAT1 activation. In this model, increase in p53 protein levels due to treatment of cells with genotoxic agents or transfection of cells with p53-expressing vectors would be sufficient to activate STAT1 by inducing the formation of a transient ATM/STAT1/p53/c-Abl1 multimeric complex that would lead to coordinate activation of c-Abl1, STAT1 and p53.

Levels of Y701-STAT1 phosphorylation induced by genotoxic agents were always much weaker than those induced by standard experimental doses of IFNs. Recent publications evidenced a temporal synergy between IFN $\alpha$ and $\gamma$ at very low doses for STAT1 activation and functional effects. ${ }^{35}$ Here, we show that genotoxic agents sensitize cells to STAT1 activation by low doses of IFN $\alpha$ and $\gamma$, with a synergistic effect for induction of the apoptotic cascade in p53-mutated cells. We also found that genotoxic agents sensitize cells to induction of IRF1 by low doses of IFN $\gamma$ (not shown). IRF1 is a transcription factor responsible for p53-independent induction of the apoptotic programme. ${ }^{15}$ These results suggest a way to overcome the resistance of p53-mutated cells to genotoxic agents by activation of the STAT1 pro-apoptotic pathway in cancers.

\section{Materials and methods}

Cell culture and reagents. PRI (Epstein Barr Virus (EBV)-transformed lymphoblastoid cell line), BL2 and BL41 (EBV-negative Burkitt lymphoma), HL60 (acute myeloid leukaemia), Jurkat (T-cell leukaemia) human cell lines were cultured in RPMI 1640 medium (Gibco BRL-Life Technologies, Cergy-Pontoise, France) supplemented with $10 \%$ tetracycline-free decomplemented fetal calf serum (Clontech, Palo Alto, CA, USA), $100 \mathrm{U} / \mathrm{ml}$ penicillin, $100 \mu \mathrm{g} / \mathrm{ml}$ streptomycin (Gibco BRL-Life Technologies), $2 \mathrm{mM}$ L-glutamine (Gibco BRL-Life Technologies), at $37^{\circ} \mathrm{C}$ in a fully humidified $5 \% \mathrm{CO}_{2}$ incubator. Plasmocyn* reagent (Cayla, Toulouse, France) was added at $50 \mu / 500 \mathrm{ml}$ to culture medium to prevent mycoplasma infection. Fludarabine* (Shering SA), actinomycin D (Sigma, St Louis, MO, USA), BFA (Sigma), Dx (Pharmachemie, Harlem, Netherlands), cisplatin (Dakota Pharm, Creteil, France), IFN $\gamma$ (Roche Diagnostics, Meylan, France) and STI571 (Novartis Pharma SA, New York, NY, USA) were used at $30 \mu \mathrm{M}, 1 \mu \mathrm{g} / \mathrm{ml}$, $40,1,30 \mu \mathrm{M}, 250 \mathrm{UI} / \mathrm{ml}$ and $1 \mu \mathrm{M}$, respectively.

Western blotting and immunoprecipitation. Western blotting was performed as described elsewhere, ${ }^{36}$ except for detection of phosphotyrosine-701 STAT1. In the latter case, sonicated cellular extracts were loaded in the gel without boiling to preserve the integrity of the phosphate residue on Y701-STAT1. Antibodies were used in the following dilutions: anti-STAT1 (9H2; Cell Signaling, Beverly, MA, USA) at 1/500, anti-phosphotyrosine-701 STAT1 (rabbit polyclonal; Cell Signaling) at 1/500, anti-p53 (DO-7 clone; Dako, Goldstrup, Denmark) at 1/500 and anti-phosphoserine-15 p53 (Santa Cruz Biotechnology, Santa Cruz, CA, USA) at 1/500, anti-MDM2 (SMP14; Dako) at 1/100, anti-phosphotyrosine-412-c-Abl1 (Cell Signaling) at 1/200, anti-phosphotyrosine-99 p73 (Cell Signaling) at 1/500, anti-p73 (Santa Cruz Biotechnology) at 1/1000, anti-phosphotyrosine-513-CD19 at 1/500, anti-CD19 (Cell Signaling) at 1/1000, anti-CD95 (C20; Santa Cruz Biotechnology) at $1 / 1000$, anti-PARP (Cell Signaling) at $1 / 500$ and anti- $\alpha$ tubulin (clone B5.2.1; Sigma) at 1/5000. The corresponding horseradish peroxidaseconjugated secondary antibody goat anti-mouse (Bio-Rad) at 1/5000 or goat antirabbit (Bio-Rad) at $1 / 5000$ was added for $45 \mathrm{~min}$. Revelation was performed by chemiluminescence (ECL; Amersham, Orsay, France) and autoradiography (Xomat R films; Sigma). For immunoprecipitation, cells were lysed in RIPA buffer (Santa Cruz Biotechnology), and 500-1000 $\mu \mathrm{g}$ was immunoprecipitated with ATM (2C1; Santa Cruz Biotechnology), p53 (DO-7 clone; Dako), c-Abl1 or STAT1 antibodies and protein A-agarose (Santa Cruz Biotechnology) overnight. The beads were washed with the lysis buffer, and the protein extract was resolved on a polyacrylamide denaturing gel.

Quantitative RT-PCR. Quantitative RT-PCR was performed as described. ${ }^{36}$ Total RNA was extracted using the Qiagen kit, following the manufacturer's recommendations. We defined, as reference RNA, a pool of RNAs extracted from different tonsils, lymph nodes and spleen with benign reactive follicular hyperplasia. RNA levels for the IFN $\gamma$ and $\alpha 2$, p21, IRF1, TAP1, p53 and MDM2 genes were quantified in parallel in the different RNA extracts and the RNA pool on an ABI PRISM 7000 Automat using the TaqManR Assay on demand TM gene expression reference system (Applied Biosystem, Foster City, CA, USA; product reference: 
Hs005174143-m1, Hs00265051-s1, Hs00355782-m1, Hs00233698-m1, Hs00388682-m1, Hs00153340-m1 and Hs00608686-m1). The c-Abl1 gene was used as a reference gene for the control of amplification (product reference: Hs00245443-m1). To validate RT-PCR quantification, expected CTs for c-Abl1 should range between 23 and 25. All amplification steps were performed in duplicate. The calculated relative gene expression level was equal to $2^{-\mathrm{DDCT}}$, where DDCT is the delta delta cycle threshold, as previously described. ${ }^{4}$

Plasmid constructs and cDNAs for p53 and MDM2 expression. The cDNAs coding for p53wt and p53 mutated on arginine 248 (p53 $3_{\mathrm{R} 248 \mathrm{Q}}$ ) were cloned from BL2 and BL41 cell lines, respectively into a Topo vector (Invitrogen, Cergy-Pontoise, France) after PCR amplification of their fulllength mRNA (p53-sense: $5^{\prime}$-GATTGGCCAGACTGCCTTC-3'; p53-antisense: $5^{\prime}$-CAAGGGTTCAAAGACCCAAA-3'). After verifying their entire sequence, these cDNAs were cloned into the Hindlll-Xbal sites of pcDNA vector (Invitrogen). The vectors coding for p53 variants, $p 53_{\mathrm{R} 156 \mathrm{P}}, \mathrm{p}_{53 \mathrm{R} 247 \mathrm{H}}, \mathrm{p} 53_{\text {del11-69, }}, \mathrm{p} 53_{\text {trunc355, }}$, $p 53_{\text {trunc338, }}$ p53 del11-69trunc338 and p53 trunc291 were established previously. ${ }^{21,22}$ The MDM2 expression vector has been described elsewhere. ${ }^{37}$

Luciferase assays. For the luciferase assay, cells were transfected with a STAT-dependent luciferase reporter construct (TAP1 promoter reporter luciferase vector (a generous gift of Dr. D Johnson)) or p53-dependent luciferase reporter construct (p21/WAF promoter reporter luciferase vector ${ }^{38,39}$ ) together with a pRLTK control vector for constitutive expression of Renilla luciferase (Promega). Cells were lysed in passive lysis buffer (Promega), and the luciferase activity of the lysates was determined using the dual luciferase reporter assay system (Promega) according to the manufacturer's instructions. Firefly luciferase activities were normalized to the activity of Renilla luciferase. Results shown are the mean of at least three experiments.

SiRNA oligonucleotides . Double-stranded RNAs (21 nt) directed against p53wt and p53 ${ }_{\text {R2480 }}$ and the corresponding control were chemically synthesized by Eurogenetec according to the previously published sequence, ${ }^{40} \mathrm{p} 53_{\mathrm{R} 248 \mathrm{WT}}: 5^{\prime}-\mathrm{GCA}$ UGAACCGGAGGCCCAUdTdT-3'; p53 22480 : 5'-GCAUGAACCAGAGGCCCAUdT dT-3'; Control 2: 5'-CAUGUCAUGUGUCACAUCUCdTdT-3'. A so-called universa control (control 1) consisting in a scramble sequence was purchased from Eurogenetec. Double-stranded siRNAs were transfected at $1.5 \mu \mathrm{g} / 5$ million cells.

Transfection of cells. Cells were transiently transfected either with expression vectors or siRNAs using the nucleofection system (Amaxa, Köln, Germany), with the V buffer, and using the T19 programme for HL60 cells and R13 programme for BL 41 cells. Where indicated, treatment of cells with the different pharmacological reagents listed above was performed $24 \mathrm{~h}$ after transfection.

Preparation of nuclear and cytosolic fractions. Nuclear and cytosolic proteins were extracted as described previously. ${ }^{4}$ Protein concentration was determined by Bradford method (Bio-Rad).

Acknowledgements. IY-M was supported by Region Limousin, Association pour la Recherche contre le Cancer and Ligue Nationale contre le Cancer. CLC was supported by Fondation pour la Recherche Médicale and Ligue Nationale contre le Cancer, comité de Haute-Vienne. UMR CNRS 6101 is supported by Ligue Nationale contre le Cancer and Cancéropôle Grand-Sud-Ouest. GWB was supported by a grant from the Wilhelm Sander-Foundation and the DFG (TRR 36). We thank Mrs. Annie Carassus and Annie Allegraud (Laboratoire d'Hématologie, CHU Dupuytren, Limoges, France) for technical assistance and Mrs. Sophie Santini (Paris, France) for English proofreading. We thank the anonymous reviewers for helping us to clarify and markedly improve the significance of our results.

1. May P, May E. Twenty years of $p 53$ research: structural and functional aspects of the $p 53$ protein. Oncogene 1999; 18: 7621-7636.

2. Stoffel A, Levine AJ. Actvation of NF-kappaB by the API2/MALT1 fusions inhibits $p 53$ dependant but not FAS induced apoptosis: a directional link between NF-kappaB and p53. Cell Cycle 2004; 3: 1017-1020

3. Townsend PA, Scarabelli TM, Davidson SM, Knight RA, Latchman DS, Stephanou A STAT-1 interacts with p53 to enhance DNA damage-induced apoptosis. J Biol Chem 2004 279: $5811-5820$
4. Baran-Marszak F, Feuillard J, Naijar I, Le Clorennec C, Bechet JM, Dusanter-Fourt I et al Differential roles of STAT1alpha and STAT1beta in fludarabine-induced cell cycle arrest and apoptosis in human B cells. Blood 2004; 104: 2475-2483.

5. Chin YE, Kitagawa M, Su WC, You ZH, Iwamoto Y, Fu XY. Cell growth arrest and induction of cyclin-dependent kinase inhibitor p21 WAF1/CIP1 mediated by STAT1. Science 1996; 272: 719-722.

6. Thomas M, Finnegan CE, Rogers KM, Purcell JW, Trimble A, Johnston PG et al. STAT1: a modulator of chemotherapy-induced apoptosis. Cancer Res 2004; 64: 8357-8364.

7. Bromberg J, Darnell Jr JE. The role of STATs in transcriptional control and their impact on cellular function. Oncogene 2000; 19: 2468-2473.

8. Marg A, Shan Y, Meyer T, Meissner T, Brandenburg M, Vinkemeier U. Nucleocytoplasmic shuttling by nucleoporins Nup153 and Nup214 and CRM1-dependent nuclear export control the subcellular distribution of latent Stat1. J Cell Biol 2004; 165: 823-833.

9. McBride KM, McDonald C, Reich NC. Nuclear export signal located within the DNA-binding domain of the STAT1transcription factor. EMBO J 2000; 19: 6196-6206.

10. McDermott U, Longley DB, Galligan L, Allen W, Wilson T, Johnston PG. Effect of p53 status and STAT1 on chemotherapy-induced, Fas-mediated apoptosis in colorectal cancer. Cancer Res 2005; 65: 8951-8960.

11. Kim HS, Lee MS. STAT1 as a key modulator of cell death. Cell Signal 2007; 19: 454-465.

12. Dimberg A, Karlberg I, Nilsson K, Oberg F. Ser727/Tyr701-phosphorylated Stat1 is required for the regulation of $\mathrm{c}-\mathrm{Myc}$, cyclins, and p27Kip1 associated with ATRA-induced G0/G1 arrest of U-937 cells. Blood 2003; 102: 254-261.

13. Kaplan DH, Shankaran V, Dighe AS, Stockert E, Aguet M, Old LJ et al. Demonstration of an interferon gamma-dependent tumor surveillance system in immunocompetent mice. Proc Natl Acad Sci USA 1998; 95: 7556-7561.

14. Ramana CV, Grammatikakis N, Chernov M, Nguyen H, Goh KC, Williams BR et al Regulation of c-myc expression by IFN-gamma through Stat1-dependent and -independent pathways. EMBO J 2000; 19: 263-272.

15. Porta C, Hadj-Slimane R, Nejmeddine M, Pampin M, Tovey MG, Espert L et al. Interferons alpha and gamma induce p53-dependent and p53-independent apoptosis, respectively. Oncogene 2005; 24: 605-615.

16. Townsend PA, Cragg MS, Davidson SM, McCormick J, Barry S, Lawrence KM et al. STAT1 facilitates the ATM activated checkpoint pathway following DNA damage. J Cell Sci2005 118 (Pt 8): 1629-1639.

17. Wade M, Wong ET, Tang M, Stommel JM, Wahl GM. Hdmx modulates the outcome of p53 activation in human tumor cells. J Biol Chem 2006; 281: 33036-33044.

18. Nelson WG, Kastan MB. DNA strand breaks: the DNA template alterations that trigger p53-dependent DNA damage response pathways. Mol Cell Biol 1994; 14: 18151823.

19. Najjar I, Baran-Marszak F, Le Clorennec C, Laguillier C, Schischmanoff O, YoulyouzMarfak I et al. Latent membrane protein 1 regulates STAT1 through NF-kappaB-dependent interferon secretion in Epstein-Barr virus-immortalized B cells. J Virol 2005; 79 4936-4943.

20. Marchini S, Ciro M, Broggini M. p53-independent caspase-mediated apoptosis in human leukaemic cells is induced by a DNA minor groove binder with antineoplastic activity. Apoptosis 1999; 4: 39-45.

21. Leblanc V, Delaunay V, Claude Lelong J, Gas F, Mathis G, Grassi J et al. Homogeneous time-resolved fluorescence assay for identifying $\mathrm{p} 53$ interactions with its protein partners, directly in a cellular extract. Anal Biochem 2002; 308: 247-254.

22. Yonish-Rouach E, Deguin V, Zaitchouk T, Breugnot C, Mishal Z, Jenkins JR et al. Transcriptional activation plays a role in the induction of apoptosis by transiently transfected wild-type p53. Oncogene 1995; 11: 2197-2205.

23. Siliciano JD, Canman CE, Taya Y, Sakaguchi K, Appella E, Kastan MB. DNA damage induces phosphorylation of the amino terminus of p53. Genes Dev 1997; 11: 3471-3481.

24. Baskaran R, Wood LD, Whitaker LL, Canman CE, Morgan SE, XU Y et al. Ataxia telangiectasia mutant protein activates $\mathrm{C}-\mathrm{Abl}$ tyrosine kinase in response to ionizing radiation. Nature 1997; $387:$ 516-519.

25. Carlesso N, Frank DA, Griffin JD. Tyrosyl phosphorylation and DNA binding activity of signal transducers and activators of transcription (STAT) proteins in hematopoietic cell lines transformed by Bcr/Abl. J Exp Med 1996; 183: 811-820.

26. Danial NN, Rothman P. JAK-STAT signaling activated by Abl oncogenes. Oncogene 2000; 19: 2523-2531.

27. Levav-Cohen Y, Goldberg Z, Zuckerman V, Grossman T, Haupt S, Haupt Y. C-Abl as a modulator of p53. Biochem Biophys Res Commun 2005; 331: 737-749.

28. Wei G, Li AG, Liu X. Insights into selective activation of p53 DNA binding by c-Abl. J Biol Chem 2005; 280: 12271-12278.

29. Ohno R. Treatment of chronic myeloid leukemia with imatinib mesylate. Int J Clin Oncol 2006; 11: 176-183.

30. Rotman G, Shiloh Y. ATM: a mediator of multiple responses to genotoxic stress. Oncogene 1999; 18: 6135-6144.

31. Yoshida K, Miki Y. Enabling death by the Abl tyrosine kinase: mechanisms for nuclear shuttling of C-Abl in response to DNA damage. Cell Cycle 2005; 4: 777-779.

32. Copland M, Jorgensen HG, Holyoake TL. Evolving molecular therapy for chronic myeloid leukaemia - are we on target? Hematology 2005; 10: 349-359.

33. Kamijo T, Weber JD, Zambetti G, Zindy F, Roussel MF, Sherr CJ. Functional and physical interactions of the ARF tumor suppressor with p53 and Mdm2. Proc Natl Acad Sci USA 1998; 95: 8292-8297. 
34. Fabbro M, Henderson BR. Regulation of tumor suppressors by nuclear-cytoplasmic shuttling. Exp Cell Res 2003; 282: 59-69.

35. Taniguchi T, Takaoka A. A weak signal for strong responses: interferon-alpha/beta revisited. Nat Rev Mol Cell Biol 2001; 2: 378-386.

36. Le Clorennec C, Youlyouz-Marfak I, Adriaenssens E, Coll J, Bornkamm GW, Feuillard J. EBV latency III immortalization program sensitizes B cells to induction of CD95-mediated apoptosis via LMP1: role of NF-kappaB, STAT1, and p53. Blood 2006; 107: 2070-2078.

37. Legube G, Linares LK, Lemercier C, Scheffner M, Khochbin S, Trouche D. Tip60 is targeted to proteasome-mediated degradation by Mdm2 and accumulates after UV irradiation. EMBO J 2002; 21: 1704-1712.
38. Drane $P$, Leblanc V, Miro-Mur F, Saffroy R, Debuire B, May E. Accumulation of an inactive form of p53 protein in cells treated with TNF alpha. Cell Death Differ 2002; 9 : 527-537.

39. Munsch D, Watanabe-Fukunaga R, Bourdon JC, Nagata S, May E, Yonish-Rouach E et al. Human and mouse Fas (APO-1/CD95) death receptor genes each contain a p53responsive element that is activated by p53 mutants unable to induce apoptosis. $J$ Biol Chem 2000; 275: 3867-3872.

40. Martinez LA, Naguibneva I, Lehrmann $H$, Vervisch A, Tchenio T, Lozano G et al. Synthetic small inhibiting RNAs: efficient tools to inactivate oncogenic mutations and restore p53 pathways. Proc Natl Acad Sci USA 2002; 99: 14849-14854. 